\title{
Time-interleaved multienergy acceleration for an x-ray free-electron laser facility
}

\author{
Toru Hara, ${ }^{1, *}$ Kenji Tamasaku, ${ }^{1}$ Takao Asaka, ${ }^{1}$ Takahiro Inagaki,,${ }^{1}$ Yuichi Inubushi, ${ }^{1}$ Tetsuo Katayama, ${ }^{2}$ Chikara Kondo, ${ }^{1}$ \\ Hirokazu Maesaka, ${ }^{1}$ Shinichi Matsubara, ${ }^{2}$ Takashi Ohshima, ${ }^{1}$ Yuji Otake, ${ }^{1}$ Tatsuyuki Sakurai, ${ }^{1}$ Takahiro Sato, ${ }^{1, \dagger}$ \\ Hitoshi Tanaka, ${ }^{1}$ Tadashi Togashi, ${ }^{2}$ Kazuaki Togawa, ${ }^{1}$ Kensuke Tono, ${ }^{2}$ Makina Yabashi, ${ }^{1}$ and Tetsuya Ishikawa ${ }^{1}$ \\ ${ }^{1}$ RIKEN SPring-8 Center, Kouto 1-1-1, Sayo, Hyogo 679-5148, Japan \\ ${ }^{2}$ Japan Synchrotron Radiation Research Institute, Kouto 1-1-1, Sayo, Hyogo 679-5198, Japan
}

(Received 24 April 2013; published 15 August 2013)

\begin{abstract}
To meet the demand from the growing number of user experiments, multi-beam-line operation with a wide spectral range is seriously considered in $\mathrm{x}$-ray free-electron laser facilities. In a conventional design, the beam line of low photon energies branches off from the middle of the accelerator to take out low energy electron beams. Here in this paper, a novel method is proposed to deliver bunch-to-bunch energy changed electron beams at the end of the accelerator. Since all accelerator components are operated in steady state, this method enables quasisimultaneous operation of multi-beam-line in the same undulator hall without degrading the stability and performance of the electron beam.
\end{abstract}

DOI: 10.1103/PhysRevSTAB.16.080701

PACS numbers: 41.60.Cr, 29.20.Ej, 29.27.-a

\section{INTRODUCTION}

After the successful operation of the Linac Coherent Light Source (LCLS) and the SPring-8 Angstrom Compact free-electron LAser (SACLA), x-ray freeelectron lasers (XFELs) have been recognized as a strong tool in diverse scientific fields [1,2], and many projects follow in coming years such as Euro-XFEL, SwissFEL, and PAL-XFEL [3-5]. Compared to current third generation synchrotron radiation facilities, XFELs provide femtosecond short $\mathrm{x}$-ray pulses with more than 10 orders of magnitude higher peak brightness. Together with the transverse coherence, these are the most significant features of XFELs.

The XFEL facility is an expensive light source due to its single-user operation. Since the storage ring of a synchrotron radiation facility recirculates the same electron bunches in the ring under an equilibrium state, many beam lines can be installed around the ring. Thus, many users can simultaneously run their experiments, namely it is a multiuser facility. On the other hand, the XFEL facility uses a linear accelerator (LINAC) in order to generate highly dense electron beams, which are required to obtain sufficiently high gain for lasing. Since the six-dimensional electron density after lasing, particularly the energy spread, is degraded and no more usable, the number of beam line is, in principle, limited to one. In order to ease this disadvantage, quasisimultaneous operation of multi-beamline using a fast switching kicker magnet is planned in

\footnotetext{
*toru@spring8.or.jp

${ }^{\dagger}$ Present address: School of Science, The University of Tokyo.
}

Published by the American Physical Society under the terms of the Creative Commons Attribution 3.0 License. Further distribution of this work must maintain attribution to the author(s) and the published article's title, journal citation, and DOI. all XFEL facilities currently in operation or under construction. Although the alternate distribution of electron bunches to each beam line results in reduction of a pulse repetition, it allows one to improve the usability of the facility. Since the x-ray optics and experimental instruments of the beam line are different depending on the photon energy, the multi-beam-line operation also contributes to enlarge the photon spectral range.

The radiation wavelength $(\lambda)$ of XFEL is determined by the electron beam energy and the undulator parameters as follows:

$$
\lambda=\frac{\lambda_{u}}{2 \gamma^{2}}\left(1+\frac{K^{2}}{2}\right)
$$

where $\lambda_{u}$ is the undulator periodic length, $K$ is the undulator deflection parameter, and $\gamma$ is the Lorenz factor of the electron beam energy. Since the tunable range of the undulator parameters, $\lambda_{u}$ and $K$, is practically limited, particularly in short-period undulators, it is necessary to change the electron beam energy, $\gamma$, to obtain a large spectral range of operation.

Considering the quasisimultaneous multi-beam-line operation with a wide spectral range, the beam energy should be changed from bunch to bunch. However, fast variation of the rf parameters easily results in unstable operation. In XFEL, the electron bunches are longitudinally compressed by a factor of around 1/1000 in order to generate highly dense electron beams. To obtain a highly compressed bunch in a magnetic chicane bunch compressor (BC), high stability is requested to rf systems both on the phase and amplitude to precisely control and linearize the energy chirp of the electron bunch. Synchronization accuracy within $100 \mathrm{fs}$ is typically required to all rf systems of LINAC. The rf stability is also important to suppress the electron beam orbit fluctuation within a sub- $\mu$ rad level inside undulators to assure interaction between electrons 
and radiation fields. Thus, the steady state operation of the rf systems is indispensable for XFELs.

In the multi-beam-line design of XFEL, the beam line of low photon energies conventionally branches off from the middle of LINAC, where the electron beam energy is still low. As shown in the facility design of SwissFEL and PAL-XFEL, this branching design requires a bypass beam transport line or an additional experimental hall for user experiments (see Refs. [4,6]).

In this paper, a novel method is described to provide bunch-to-bunch energy changed electron beams at the end of LINAC without spoiling the stability of the rf system and the electron beam [proposed by one of the authors (Tamasaku)]. This method is applicable to any LINAC operated using pulsed rf sources. In this method, the number of the rf units effectively used for acceleration is adjusted from bunch to bunch with maintaining the stability of the rf systems. This method not only increases the flexibility of the XFEL operation but also permits one to install beam lines of a wide photon energy range in the same experimental hall.

\section{TIME-INTERLEAVED MULTIENERGY OPERATION}

Except for superconducting LINACs, XFEL is generally operated in a pulsed mode using a normal conducting LINAC at a repetition around $100 \mathrm{~Hz}$. In order to achieve high peak currents, all $\mathrm{rf}$ units of LINAC are required to work in a synchronous manner, and for that a precise timing system has been developed and equipped in XFEL facilities [7]. The timing system of XFEL generally distributes trigger and reference rf clock signals to each rf unit operated in a pulsed mode. The trigger signal, which starts charging the high voltage of each rf unit, is generated based on a power line frequency. The reference rf clock and a counter module are used to suppress temporal jitter and to adjust time delay. In SACLA, the counter module is installed in each rf unit as shown in Fig. 1 to enable the operation of specific rf units at subharmonics of the trigger frequency.

An example of the accelerator setup is shown in Fig. 1. In this example, the energy gain in the accelerating structures of one rf unit is assumed to be $200 \mathrm{MeV}$, and $60 \mathrm{pps}$ electron bunches are accelerated up to $6.8 \mathrm{GeV}$ in the upstream part of LINAC. By changing the trigger frequencies of the last six rf units as shown in Fig. 1, half of the electron bunches pass through these rf units without acceleration, so the final beam energy stays at $6.8 \mathrm{GeV}$. A quarter of the bunches are accelerated up to $7.6 \mathrm{GeV}$ by the four accelerating structures working at $30 \mathrm{~Hz}$. The last quarter of the bunches are further accelerated by the last two structures working at $15 \mathrm{~Hz}$, so the final beam energy reaches $8.0 \mathrm{GeV}$. Combining with the synchronized operation of a bunch-to-bunch switching kicker magnet, the electron beam energies of each beam line can be varied. In Fig. 1, the electron bunches of $8,6.8$, and $7.6 \mathrm{GeV}$ are alternately provided to three beam lines (BL1-3). The pulse repetition rates of these beam lines become 15, 30, and 15 pps at BL1, BL2, and BL3, respectively.

Although the pulse repetition of BL2 is twice higher than other BLs in Fig. 1, it is also possible to evenly distribute the electron bunches to three beam lines by adjusting the subharmonic frequencies and phases. By changing the acceleration gradient, the beam energies are finely adjustable. Since all rf units operate under steady condition without any fast parameter variation from bunch to bunch, stable operation of LINAC can be expected.

\section{DEMONSTRATION EXPERIMENTS USING SACLA BL3}

The bunch-to-bunch multienergy operation was examined and demonstrated at SACLA without switching the beam lines, because the XFEL undulators $\left(\lambda_{u}=18 \mathrm{~mm}\right)$ are currently installed only at BL3 and a fast kicker magnet has not been equipped yet so far. Figure 2 is a schematic layout of the SACLA facility. To generate highly dense electron beams, the accelerating structures upstream of $\mathrm{BC} 3$ are operated at off-crest rf phases for longitudinal compression of the electron bunch [8]. After the final bunch compression at BC3, the electrons are further accelerated up to the nominal beam energy by on-crest acceleration in the C-band main accelerators downstream of BC3. The SACLA undulator hall is capable to accommodate five undulator beam lines, and the distribution of the electron bunches using a fast switching kicker magnet is planned at the end of LINAC. Currently, a dc bending

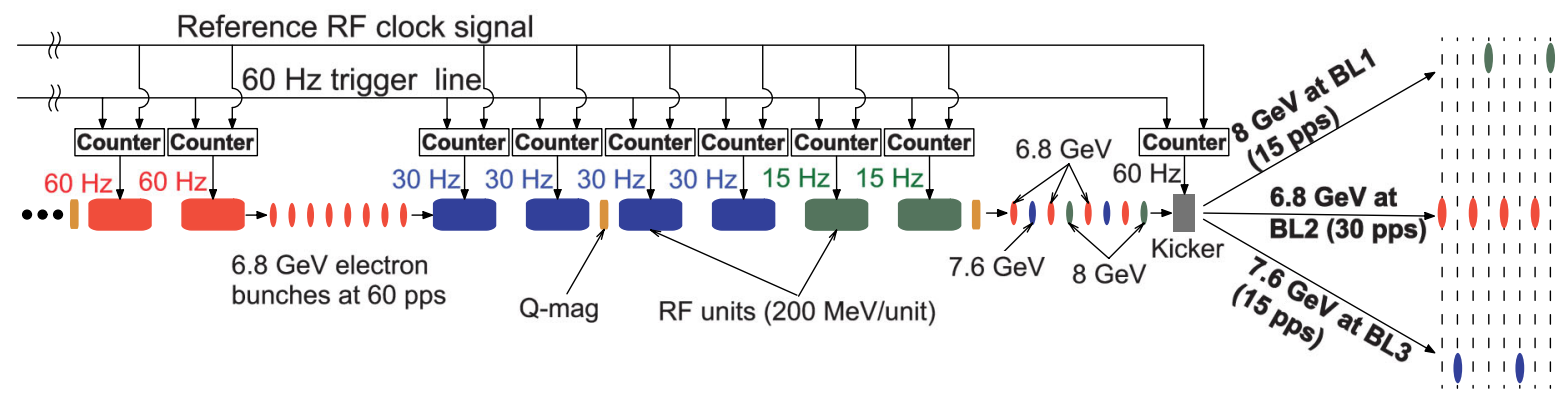

FIG. 1. An example of accelerator setup for multienergy operation. 

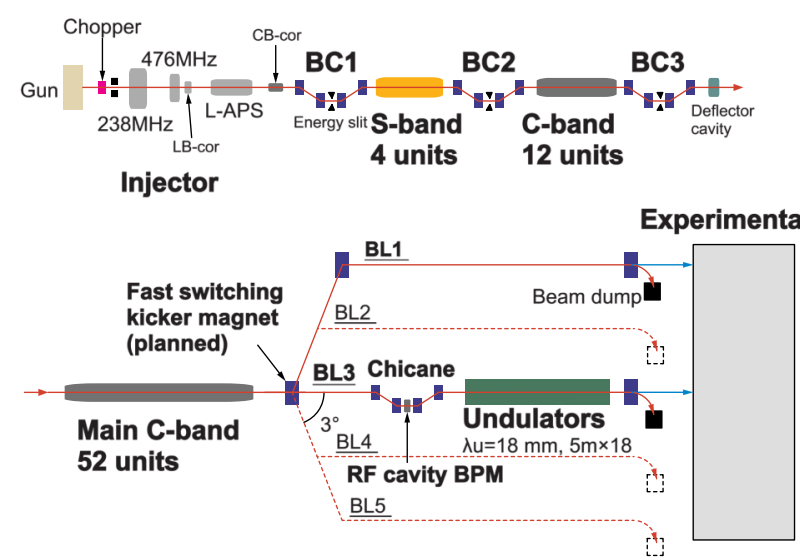

FIG. 2. Schematic view of the SACLA facility. Currently a dc bending magnet is installed at the location of the fast switching kicker magnet.

magnet is installed instead of a kicker magnet to switch beam lines between BL1 and BL3.

In the demonstration, the maximum beam energy and repetition of the electron bunch were set at $8 \mathrm{GeV}$ and $10 \mathrm{pps}$, respectively. Thus, the trigger frequency of the rf units was $10 \mathrm{~Hz}$ except for the last eight rf units in the C-band main accelerators, which were operated at $5 \mathrm{~Hz}$. Since the beam energy gain in one C-band rf unit, which drives two accelerating structures, is about $130 \mathrm{MeV}$, the beam energy was expected to be alternately changed by $1.1 \mathrm{GeV}$. The maximum beam energy separation is limited to $1.1 \mathrm{GeV}$ to avoid hitting the beam to the duct wall after a $\mathrm{dc}$ bending magnet of the beam dump. The beam energies were measured at a dispersive chicane installed upstream of the BL3 undulators (Fig. 2), where the horizontal beam position was measured using an rf cavity-type beam position monitor (RF-BPM) [9]. The measured bunch-to-bunch beam energies are shown in Fig. 3, in which the electron beam energy is alternately changed between 8.0 and $6.9 \mathrm{GeV}$.

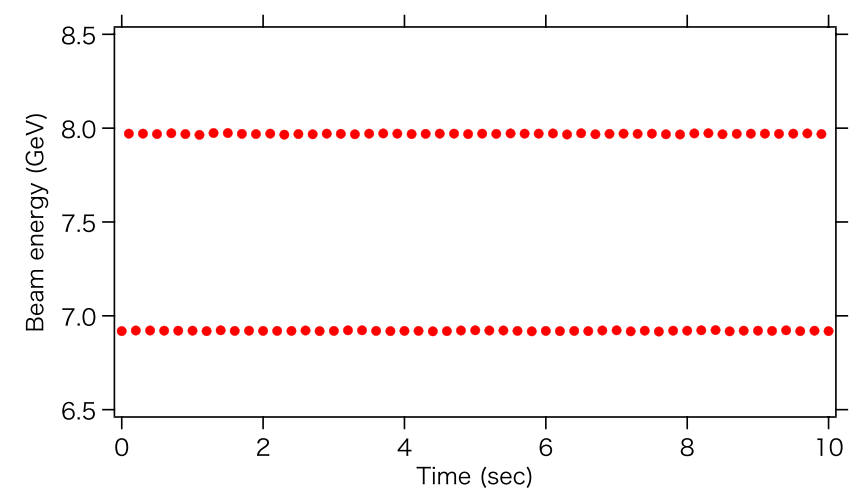

FIG. 3. Beam energies measured at the dispersive chicane of BL3. The bunch repetition was 10 pps and the beam energy was alternately changed between 8.0 and $6.9 \mathrm{GeV}$.
The stabilities of the electron beam energy and orbit were also measured by the RF-BPMs in this two-energy operation. The standard deviations of the relative beam energy variation during 30 seconds were $2.7 \times 10^{-4}$ and $2.5 \times 10^{-4}$ at 6.9 and $8.0 \mathrm{GeV}$, respectively. These values are equivalent to $2.7 \times 10^{-4}$, which is the stability of the single-energy operation at $6.9 \mathrm{GeV}$. The beam orbit fluctuations were calculated by multiplying the standard deviations of the measured beam position and angle using two RF-BPMs at the undulator entrance. The results were $0.97 \mathrm{pm}$-rad (horizontal) and $0.77 \mathrm{pm}-\mathrm{rad}$ (vertical) at $6.9 \mathrm{GeV}$, and $1.1 \mathrm{pm}-\mathrm{rad}$ (horizontal) and $0.17 \mathrm{pm}-\mathrm{rad}$ (vertical) at $8.0 \mathrm{GeV}$, respectively. These values are comparable to $0.81 \mathrm{pm}$-rad (horizontal) and $0.82 \mathrm{pm}-\mathrm{rad}$ (vertical) of the single-energy operation at $6.9 \mathrm{GeV}$.

Since quadrupole and steering magnets are not adjusted with the beam energy in this multienergy operation, there might be concerns about the energy dependent deviations of the transverse envelope and orbit of the electron beams. In order to minimize them, the alignment of accelerating structures and the magnetic shielding for ambient fields should be considered. However, these deviations do not crucially prevent the XFEL operation, because the deviations of the transverse beam envelope and orbit in LINAC rarely cause the degradation of beam quality in practice unless in extreme cases. Once the electron bunches are distributed to each beam line by a switching kicker magnet, these energy dependent deviations can be corrected and it is possible to match the beam envelope and orbit to the periodic lattice of undulators.

For example, the beam envelopes are shown in Fig. 4, which are calculated for 7.8 and $4 \mathrm{GeV}$ beams using the focusing optics of SACLA [10]. Quadrupole magnets are placed every four rf units $(16 \mathrm{~m})$ in the C-band main accelerator to compose a FODO (alternate focusing and defocusing) lattice. The quadrupole fields are initially optimized for the $7.8 \mathrm{GeV}$ beam of BL2 [Fig. 4(a)]. Then the envelope is recalculated for the $4 \mathrm{GeV}$ beam of BL3 [Fig. 4(b)] with keeping the quadrupole fields of the main accelerator the same. The beam envelope starts to deviate from FODO in the last half of the C-band main accelerator, where the rf units are switched off. However, this distortion can be corrected upstream of BL3 and matched to the undulator lattice. In a similar way, the injection beam orbits to the undulators can also be corrected at each beam line after the switching kicker magnet. The range of the multienergy operation is limited by the envelope and orbit distortions, which vary depending on the accelerator parameters and the focusing optics. In the case of SACLA, we expect the maximum range of the multienergy operation to be between 4 and $8.5 \mathrm{GeV}$.

Since there is currently one operational beam line (BL3) in SACLA, the beam envelope and orbit cannot be corrected for multienergy beams. Even under slightly 

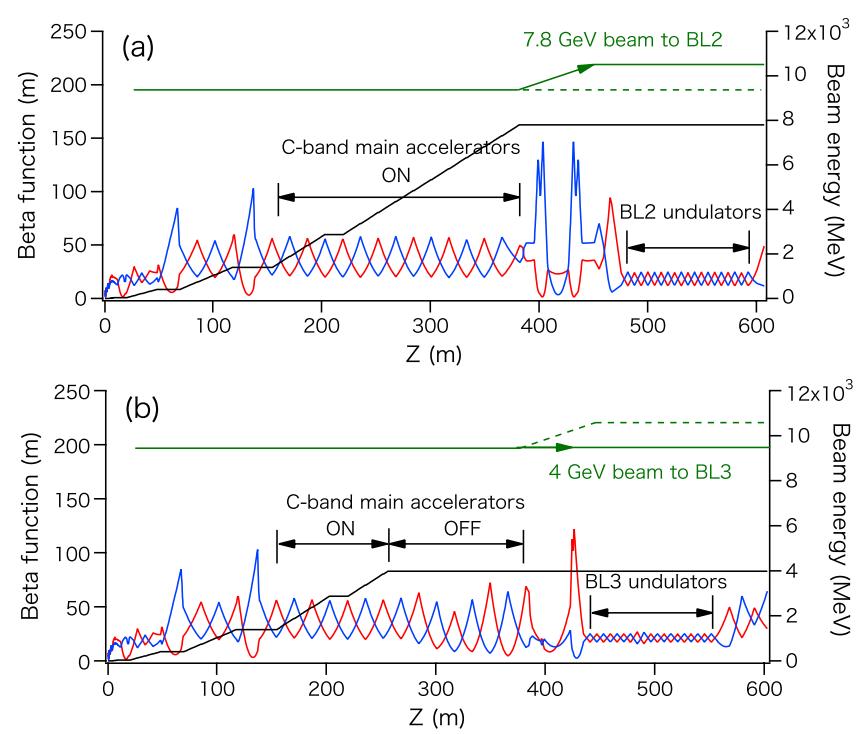

FIG. 4. Beam envelopes calculated for 7.8 and $4 \mathrm{GeV}$ beams with the focusing lattice of SACLA. The lattice is initially optimized for the $7.8 \mathrm{GeV}$ beam of BL2 (a), then the envelope distortion is calculated for the $4 \mathrm{GeV}$ beam of BL3 with the same quadrupole fields at the C-band main accelerator (b). The red and blue lines are horizontal and vertical beta functions, and the black lines are the beam energy along the accelerator. The locations of the C-band main accelerator and the undulators are indicated in the figures. Note that there is a $16 \mathrm{~m}$ long drift space in the $\mathrm{C}$-band main accelerator around $Z=$ $200 \mathrm{~m}$.

mismatched conditions, however, self-amplified spontaneous emission (SASE) was obtained in the two-energy operation of a $500 \mathrm{MeV}$ energy separation. Figure 5 shows the SASE spectrum at two wavelengths emitted from the same BL3 undulators with $K=2.1$. The repetition of the electron beam was $10 \mathrm{pps}$ and four rf units were operated at $5 \mathrm{~Hz}$ in the C-band main accelerators. Therefore the beam energy was alternately changed between 7.3 and $7.8 \mathrm{GeV}$. The spectrum was measured by scanning a monochromator,

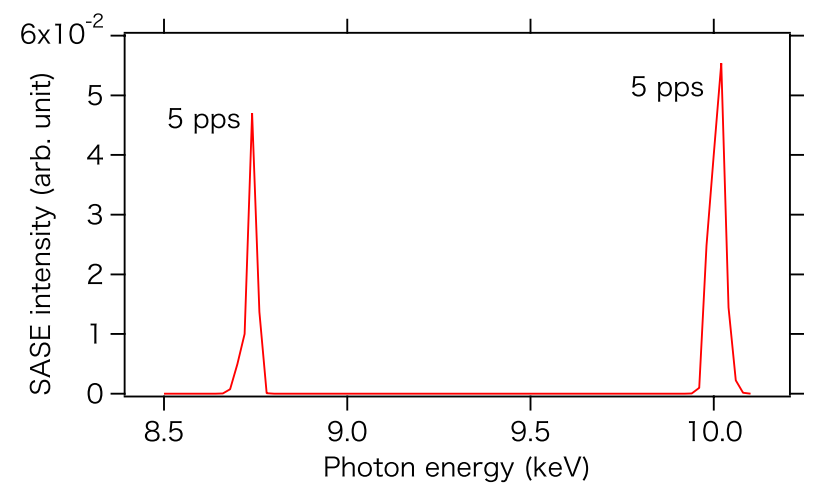

FIG. 5. Spectra of SASE measured by scanning a monochromator. The electron bunch repetition was $10 \mathrm{pps}$ and the beam energy was alternately changed between 7.3 and $7.8 \mathrm{GeV}$.

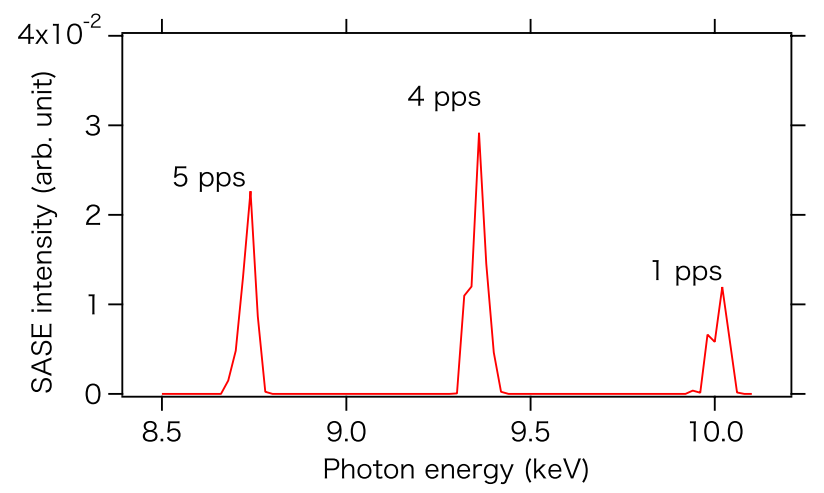

FIG. 6. Spectra of SASE measured by scanning a monochromator. The electron bunch repetition was $10 \mathrm{pps}$ and the beam energy was changed between $7.3,7.55$, and $7.8 \mathrm{GeV}$.

so it is averaged over pulses. The electron beam envelope and orbit were first optimized at $7.8 \mathrm{GeV}$ and the photon pulse energy of $10 \mathrm{keV}$ was about $200 \mu \mathrm{J}$ in the single-energy operation. In the two-energy operation of Fig. 5, the same pulse energy was obtained at $10 \mathrm{keV}$, which proves that the quality and stability of the electron beam were maintained the same level and good enough for lasing.

The number of beam energies was extended to three in Fig. 6. The electron beam repetition was $10 \mathrm{pps}$ with $K=2.1$. In this case, two rf units were operated at $5 \mathrm{~Hz}$ and the other two units at $1 \mathrm{~Hz}$. Consequently, the repetition rates of the photon pulses became 1,4 , and 5 pps at $10,9.35$, and $8.7 \mathrm{keV}$, respectively.

\section{DISCUSSIONS}

In the demonstration, the maximum beam energy separation was limited to $1.1 \mathrm{GeV}$. However, the energy separation can be increased unless the deviations of the transverse electron beam envelope and orbit become unacceptably large in the accelerator.

The proposed method in this paper achieves quasisimultaneous multi-beam-line operation with different beam energies in the same experimental hall, which allows one to improve the usability and to enlarge the spectral range of XFEL. Compared to a branching-off design, the wakefields of accelerating structures can be used to correct the energy chirp of low energy electron beams. Thus, a dechirping device, such as a corrugated pipe, may not be necessary [11]. By controlling the trigger to each rf unit, this method can be applied to any XFEL facilities to generate multienergy beams without deteriorating the quality and stability of the electron beam.

\section{ACKNOWLEDGMENTS}

The authors wish to acknowledge the support of the SACLA operation team, helping the accelerator operation and data analyses. 
[1] P. Emma et al., Nat. Photonics 4, 641 (2010).

[2] T. Ishikawa et al., Nat. Photonics 6, 540 (2012).

[3] M. Altarelli et al., DESY Report No. 2006-097, 2007.

[4] J. H. Han, H. S.Kang, and I. S. Ko, in Proceedings of the 3rd International Particle Accelerator Conference, New Orleans, Louisiana, USA, 2012 (IEEE, Piscataway, NJ, 2012), p. 1735.

[5] Romain Ganter, SwissFEL Conceptual Design Report, Villigen, V19, 2011.

[6] S. Reiche, in Proceedings of the 33rd International Free Electron Laser Conference, Shanghai, 2011 (SINAP, Shanghai, China, 2011), p. 223 [http://accelconf.web.cern .ch/AccelConf/FEL2011/index.htm].
[7] Y. Otake, T. Ohshima, N. Hosoda, H. Maesaka, T. Fukui, M. Kitamura, and T. Shintake, Nucl. Instrum. Methods Phys. Res., Sect. A 696, 151 (2012).

[8] K. Togawa, T. Hara, and H. Tanaka, Phys. Rev. ST Accel. Beams 12, 080706 (2009).

[9] H. Maesaka, H. Ego, S. Inoue, S. Matsubara, T. Ohshima, T. Shintake, and Y. Otake, Nucl. Instrum. Methods Phys. Res., Sect. A 696, 66 (2012).

[10] T. Hara, K. Togawa, and H. Tanaka, Nucl. Instrum. Methods Phys. Res., Sect. A 624, 65 (2010).

[11] K. L.F. Bane and G. Stupakov, Nucl. Instrum. Methods Phys. Res., Sect. A 690, 106 (2012). 